\title{
Self-breaking in planar few-atom Au constrictions for nanometer-spaced electrodes
}

\author{
K. O'Neill, ${ }^{\text {a) }}$ E. A. Osorio, and H. S. J. van der Zant \\ Kavli Institute of Nanoscience Delft, Delft University of Technology, Lorentzweg 1, 2628 CJ Delft, \\ The Netherlands
}

(Received 16 November 2006; accepted 20 February 2007; published online 28 March 2007)

\begin{abstract}
The authors present results on electromigrated Au nanojunctions broken near the conductance quantum of $77.5 \mu \mathrm{S}$. At room temperature it is found that wires, initially narrowed by an actively-controlled electromigration technique down to a few conductance quanta, continue to narrow after removing the applied voltage. Separate electrodes form as mobile gold atoms continuously reconfigure the constriction. They find, from results obtained on over 300 samples, no evidence for gold cluster formation in junctions broken without an applied voltage, implying that gold clusters may be avoided by using this self-breaking technique. (C) 2007 American Institute of Physics. [DOI: 10.1063/1.2716989]
\end{abstract}

Electronic devices based on single nanometer-sized molecules show promising routes to exploiting the functionality available through organic synthesis. In addition such devices provide an experimental platform to understand the electronic, ionic, and mechanical degrees of freedom of a single molecule and its coupling to the environment. While many methods exist to create nanometer-spaced electrodes, the presence of a gate electrode is crucial to correctly identify the signatures of single-molecule conduction at low temperature, implying that a planar geometry is required. In this geometry, a common method for creating the nanometersized electrode spacing is by electromigration, in which a large current density of $\sim 10^{8} \mathrm{~A} \mathrm{~cm}^{-2}$ is used to deform a small gold wire until physically separated electrodes are formed. ${ }^{1}$ It has remained a persistent challenge to unambiguously determine the presence of a trapped single molecule in transport measurements using the electromigration technique. Transport measurements are hindered by the uncontrolled nature of the breaking process, which produces nanogaps of a wide range of sizes, and the formation of gold clusters that give signatures of Coulomb blockade and Kondo physics, unrelated to conduction processes through single molecules. ${ }^{2,3}$

This letter demonstrates a "self-breaking" effect in gold wires that are narrowed by electromigration to a few atoms. Gold nanoconstrictions fabricated at room temperature tend to be unstable; on a time scale of tens of minutes or hours they break further until the conductance reaches values $\ll 100 \mu \mathrm{S}$ without an applied voltage. Subsequent measurements at low temperature show that self-broken wires are less likely to produce gold clusters than samples that are actively broken into separate electrodes. This self-breaking effect has also been observed in transmission electron microscope studies, ${ }^{4}$ indicating that the decreasing conductance is due to a physical separation of the electrodes in time.

Samples are fabricated as follows: thin wire bridges, with a cross section of $100 \times 12 \mathrm{~nm}^{2}$ and a typical length of $500 \mathrm{~nm}$ are evaporated over a gate dielectric. The gates are formed using aluminium wires and its native aluminium oxide of a few nanometers is used as the gate dielectric. Con-

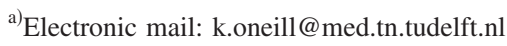

tact to the bridge is made by large wires which contribute a small series resistance. ${ }^{5}$ The resistance between bonding pads before the wires are narrowed is typically $100-200 \Omega$. To initially narrow the gold wires by electromigration, we use an active breaking scheme, ${ }^{6}$ similar to the ones previously reported. ${ }^{7}$ In the active breaking process, the voltage is increased from below the electromigration threshold $(>200 \mathrm{mV})$ while sampling the current; the maximum current is $\sim 8 \mathrm{~mA}$. If the absolute resistance of the wire increases by a value determined during the sweep, typically around $10 \%$, the applied voltage is reduced back to $100 \mathrm{mV}$, and the sweep is repeated with a new value for the wire resistance. With this method, nanoconstrictions may be narrowed to a target conductance with high reproducibility, often within $10 \%$, provided the target conductance is greater than the conductance quantum $G_{0}=2 e^{2} / h=77 \mu \mathrm{S}$. Electromigration events change the junction resistance on a time scale $<100 \mu \mathrm{s}$, and the rate typically chosen for voltage output and current sampling is $\sim 25-50 \mu$ s. Figure 1(a) shows atomic force microscope images of a sample before (I) and after (II) narrowing using this technique. We note the formation of a hillock downstream of the gap, demonstrating that electron wind force is responsible for the transfer of momentum between electrons and gold atoms.

While active breaking allows the resistance of a constriction to be precisely controlled, the mobility of gold at room temperature is high enough to break the wire completely, resulting in two separate electrodes, even without applying a bias. Figures 1(b)-1(d) show the conductance versus time of three wires at room temperature when initially narrowed by active breaking to $4 \mathrm{k} \Omega, 2 \mathrm{k} \Omega$, and $900 \Omega$. Here, we plot the numerical $d I / d V$ around zero bias obtained by sweeping the applied voltage from -100 to $100 \mathrm{mV}$ while measuring the current through the constriction, at a rate of $1 \mathrm{sweep} / \mathrm{s}$. The sample in Fig. 1(e) was narrowed first to $700 \Omega$ and then measured with a lock-in amplifier using an oscillation of $1 \mathrm{mV}_{\mathrm{RMS}}$ around zero bias. To emphasize that self-breaking occurs even in the absence of an applied electric field, we have carried out several hundreds of experiments in which no bias is applied after narrowing, and have observed selfbreaking in all cases. 
(a)

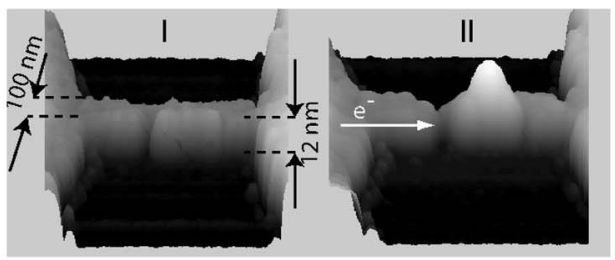

(b)

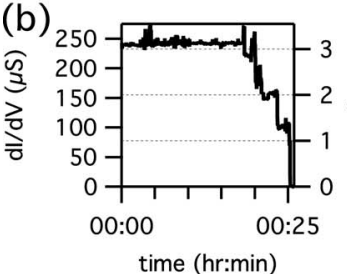

(c) $500-6$
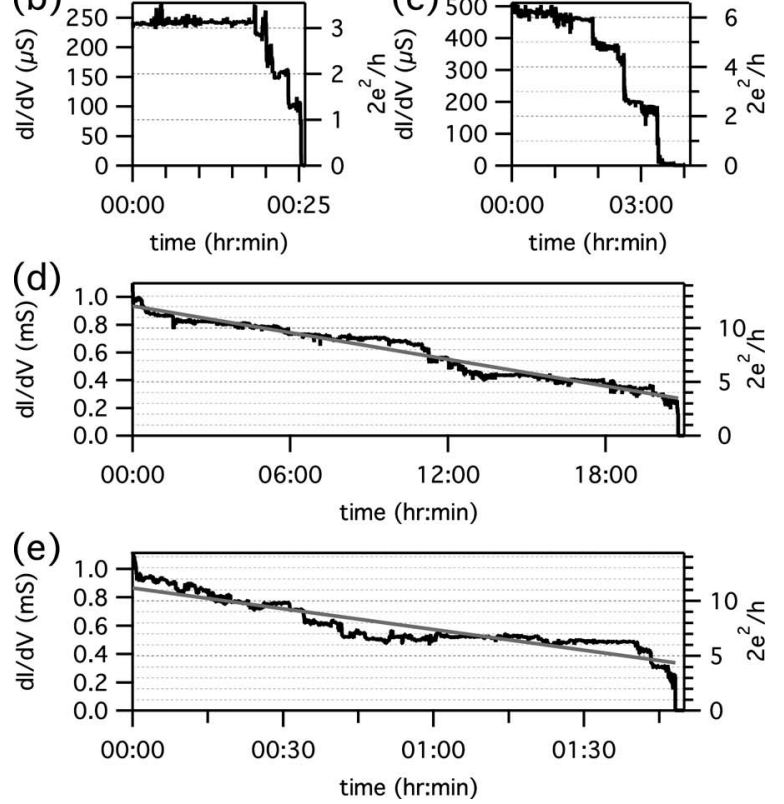

FIG. 1. (a) Atomic force microscope images of a sample before and after narrowing. (b)-(e) show self-breaking data in which conductance (in $S$ on the left axis and conductance quanta $2 e^{2} / h$ on the right axis) is plotted against time (in hours and minutes), measured at room temperature and in a pressure less than $10^{-5}$ Torr. The straight lines in (d) and (e) are fits up to the points near the conductance $2 G_{0}$.

In each sample, the resulting reduction in conductance is not continuous but evolves in discrete steps. Configurations may persist for long times before changing, and the time to reach less than one conductance quantum may vary between tens of minutes, to tens of hours. The rate of conductance drop until $2 G_{0}$ is $\sim 2000 \mathrm{~s} / G_{0}$ for Figs. $1(\mathrm{~b})$ and $1(\mathrm{c})$, $8700 \mathrm{~s} / G_{0}$ for Fig. $1(\mathrm{~d})$, and $1000 \mathrm{~s} / G_{0}$ for Fig. 1(e). We note that, as the junction approaches a conductance of $\sim 1 G_{0}$, the junctions are typically only stable for a few seconds. This behavior corresponds to the removal of the final atom between the two electrodes until charge can only be transferred by tunneling processes.

Despite the unstable nature of the few-atom constrictions at room temperature, fast acquisition allows $I-V$ 's to be recorded as the system evolves. Figure 2(a) shows a selection of the $d I / d V$ vs $V$ curves collected during self-breaking of the sample presented in Fig. 1(c) at $300 \mathrm{~K}$, computed from the $I$ - $V$ curves as previously described. As we have demonstrated above, wires narrowed at room temperature are unstable, further characterization may be performed at low temperature. In contrast to room temperature, gold wires narrowed by active breaking at $1.6 \mathrm{~K}$ are stable, and their $I-V$ characteristics do not vary in time. In this conductance range, constrictions of a specific target resistance can be created by actively controlled electromigration to within 5\%, even in the conductance range near $1 G_{0}$. The lowtemperature data in Fig. 2(b) were collected with a lock-in amplifier in the order indicated by the arrow. We see that, in

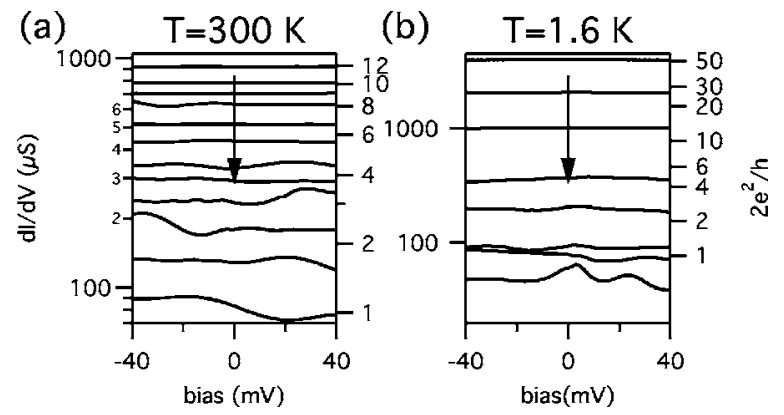

FIG. 2. Differential conductance curves for constrictions of varying conductance as a function of bias voltage, at room temperature and low temperature. The room temperature data are recorded during the breaking process. For the low-temperature data each curve represents a different configuration obtained with active breaking. (The arrows indicate the sequence in which the data were collected).

both the unstable room temperature constrictions, and in the stable low-temperature constrictions, the $d I / d V$ - $V$ curves are flat for $d I / d V \geqslant 5 G_{0}$, and become highly nonlinear near $1 G_{0}$. In addition, measurements of the samples narrowed at low temperature to $d I / d V \sim 1 G_{0}$ at $1.6 \mathrm{~K}$ reveal negligible magnetic field dependence up to $10 \mathrm{~T}$. For electron interference to be the origin of the observed oscillations, loops of $20 \mathrm{~nm}$ or less are implied. ${ }^{8}$

Finally, we present statistics on samples broken at room temperature with single organic molecules either by breaking the wires with a self-assembled monolayer or by breaking directly in a solution of the molecules of interest, collected over approximately a year. ${ }^{9}$ After breaking, samples were cooled to $1.6 \mathrm{~K}$ and the current was measured as a function of gate and bias for each device. Of 162 samples broken by narrowing the constriction to a few atoms wide and then allowed to self-break with no bias applied, 24 showed gate dependence, and none showed indications of transport through gold clusters (see below). In contrast, of 171 samples broken by active breaking into the tunneling regime (applying above-threshold biases until $>100 \mathrm{k} \Omega$ ), 38 show gate dependence, of which six samples show indications of transport through gold clusters.

In discriminating between transport through gold clusters and transport through single molecules we identify two parameters of a double-tunneling system with a single charging island: the electron addition energy and the coupling to the gate electrode ${ }^{10} \mathrm{We}$ attribute charging energies less than $100 \mathrm{meV}$ in combination with a gate coupling greater than 0.2 to gold grains. We reach these parameters as follows: by identifying single-molecule samples through their vibrational spectra ${ }^{11}$ it has been established that for the molecules studied here, electron addition energies typically lie in a range greater than $100 \mathrm{meV}$, comparable with electron addition energies measured using other techniques. ${ }^{12}$ In addition, transport that is due to single molecules has consistently shown a low gate coupling, not larger than 0.15 . Gold clusters, on the the other hand, would be in direct contact with the gate dielectric, and so would be expected to have a higher gate coupling, which we typically find to be $0.25 .^{13}$

We have also observed self-breaking in narrowed gold wires on silicon oxide substrates, instead of aluminium gates, revealing comparable results to those presented here. In addition, experiments with platinum wires have shown no selfbreaking effect, indicating that the effect is connected with some intrinsic property of the wire material. The selflicense or copyright; see http://apl.aip.org/about/rights_and_permissions 


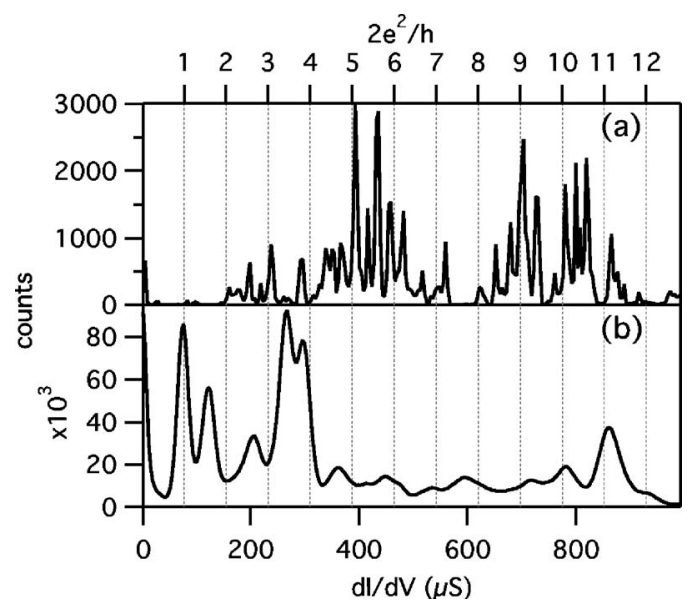

FIG. 3. (a) Conductance histogram constructed from data presented in Fig. 1; (b) conductance histogram constructed from eight samples measured with a sampling rate of $100 \mu \mathrm{s}$, broken under a applied potential of $225 \mathrm{mV}$; each graph uses 20 bins per $\mathrm{G}_{0}$.

breaking of narrowed wires can be compared with diffusion of gold, as observed in scanning tunneling microscope (STM) experiments. ${ }^{14}$ Here the surface diffusion velocities were measured in the range from 0.03 to $0.2 \AA / s$. Recalculating this as the time required to jump the nearest neighbor distance of $2.88 \AA$, surface mobility implies that it takes 10-100 s for gold atoms to move in and out of a contact, contributing to or reducing the conductance by $G_{0}$. This is within an order of magnitude faster than the observed rate shown in Fig. 1 for conductances $>2 G_{0}$, and an order of magnitude slower than for junctions with conductance $<2 G_{0}$. Given these variations, there is rough agreement between this crude calculation and the measurements which seems to suggest that a diffusion mechanism drives the selfbreaking process.

The traces in Figs. 1(b) and 1(c) seem to imply a preference for conductances at integer multiples of $G_{0}$, consistent with previous observations. ${ }^{7}$ Figure 3(a) shows a histogram in which the occurance of conductances is plotted in a histogram, using the data presented in Figs. 1(b)-1(d). Figure 3(b) shows a similar histogram of conductance values built from eight samples with a driving field close to the electromigration threshold, using a much higher sampling rate. It is clear that, while some of the conductance peaks fall on integer multiples of $G_{0}$, many others do not, and it is therefore difficult to conclude that preferred conductances exist. For comparison, STM (Ref. 15) and mechanically controllable break junction experiments show that at least $\sim 230$ traces have to be considered, implying that the statistics in our experiments may be too low. In addition, in such experiments only the first three conductance peaks are visible, for which we obtain fewer statistics.

The cause of the nonlinear $d I / d V$ vs $V$ curves shown in Fig. 2 is unclear. An explanation for the effect may come from electron interference in ballistic atomic-scale junctions, ${ }^{16}$ which may be of the same order of magnitude as the measurements presented here. Further measurements, at room and low temperatures, have not revealed a clear suppression of the nonlinearities near integer multiples of $G_{0}$, which would strongly corroborate the electron interference theory. An alternative cause could stem from contamination in the junction. STM experiments establish that contamination by air (notably water vapor) both modifies the surface of gold samples ${ }^{14}$ and changes the second derivative $G^{\prime \prime}$ of one and two atom junctions. ${ }^{17}$ We believe that this is unlikely to explain the variation in $G^{\prime \prime}$ observed in our junctions, which is $\sim 1000$ times higher than reported values (Ref. 17).

Financial support was obtained from the Dutch organization for Fundamental Research on Matter (FOM), and the "Nederlandse Organisatie voor Wetenschappelijk Onderzoek" (NWO). The authors thank M. Poot for the data on silicon oxide substrates. One of the authors (K.O.'N.) was supported by the Marie Curie Fellowship organization. The authors are grateful to A. Ito, T. Bjørnholm, and M. Ruben for providing the molecules.

${ }^{1}$ H. Park, A. K. L. Kim, A. P. Alivisatos, J. Park, and P. L. McEuen, Appl. Phys. Lett. 75, 301 (1999).

${ }^{2}$ J. I. Gonzalez, T.-H. Lee, M. D. Barnes, Y. Antoku, and R. D. Dickson, Phys. Rev. Lett. 93, 147402 (2004); R. Sordan, K. Balasubramanian, and K. Kern, Appl. Phys. Lett. 87, 013106 (2005).

${ }^{3}$ H. Heersche, Z. de Groot, J. A. Folk, L. P. Kouwenhoven, H. S. J. van der Zant, A. A. Houck, J. Labaziewicz, and I. L. Chuang, Phys. Rev. Lett. 96, 017205 (2006).

${ }^{4}$ D. R. Strachan, D. E. Smith, M. D. Fischbein, D. E. Johnson, B. S. Guiton, M. Drndić, D. A. Bonnell, and A. T. Johnson, Jr., Nano Lett. 6, 441 (2006); H. Heersche, G. Lientschnig, K. O’Neill, H. S. J. van der Zant, and $\mathrm{H}$. W. Zandbergen (unpublished).

${ }^{5}$ M. Trouwborst, S. J. van der Molen, and B. J. van Wees, J. Appl. Phys. 99, 114316 (2006).

${ }^{6}$ H. S. J. van der Zant, Y.-V. Kervennic, M. Poot, K. O’Neill, Z. de Groot, H. B. Heersche, N. Stuhr-Hansen, T. Bjørnholm, D. Vanmaekelbergh, C. A. van Walree, and L. W. Jenneskens, Faraday Discuss. 131, 347 (2006). ${ }^{7}$ D. R. Strachan, D. E. Smith, D. E. Johnston, T.-H. Park, M. J. Therien, D. A. Bonnell, and A. T. Johnson, Appl. Phys. Lett. 86, 043109 (2005); A. A. Houck, J. Labaziewicz, E. K. Chan, J. A. Folk, and I. L. Chuang, Nano Lett. 5, 1685 (2005).

${ }^{8}$ Assuming magnetic flux $h / e$ enclosed by circular interfering electron paths in a magnetic flux density of $10 \mathrm{~T}$.

${ }^{9}$ Oligophenylenevinylene 3 , oligophenylenevinylene 5 , spiro-fused triarylamine, $\left[\mathrm{Co}_{4}^{\mathrm{II}} L_{4}\right]^{8+}[2 \times 2]$-grid-type complexes, Co-terpyridine.

${ }^{10}$ K. Likharev, Proc. IEEE 87, 606 (1999).

${ }^{11}$ E. A. Osorio, K. O'Neill, N. Stuhr-Hansen, O. F. Nielsen, T. Bjørnholm, and H. S. J. van der Zant, Adv. Mater. (Weinheim, Ger.) 19, 281 (2007).

${ }^{12}$ S. Kubatkin, A. Danilov, M. Hjort, J. Cornil, J.-L. Brédas, N. StuhrHansen, P. Hedegård, and $\mathrm{T}$. $\mathrm{Bj}^{\circ}$ rnholm, Nature (London) 425, 698 (2003).

${ }^{13}$ H. S. J. van der Zant, E. A. Osorio, M. Poot, and K. O’Neill, Phys. Status Solidi B 248, 3408 (2006); K. I. Bolotin, F. Kuemmeth, A. N. Pasupathy, and D. C. Ralph, Appl. Phys. Lett. 84, 3154 (2004).

${ }^{14}$ C. Roberts, B. Hoffmann-Millack, and W. S. Steer, J. Vac. Sci. Technol. B 9, 841 (1990).

${ }^{15}$ M. Brandbyge, J. Schiøtz, M. R. Sorenson, P. Stoltze, K. W. Jacobsen, J. K. N ${ }^{\circ}$ rskov, L. Olesen, E. Laegsgaard, I. Stensgaard, and F. Besenbacher, Phys. Rev. B 52, 8499 (1995).

${ }^{16}$ B. Ludoph, M. H. Devoret, D. Esteve, C. Urbina, and J. M. van Ruitenbeek, Phys. Rev. Lett. 82, 1530 (1999); C. Untiedt, G. Rubio Bollinger, S. Vieira, and N. Agraït, Phys. Rev. B 62, 9962 (2000).

${ }^{17}$ K. Hansen, S. K. Nielsen, M. Brandbyge, E. Laegsgaard, I. Stensgaard, and F. Besenbacher, Appl. Phys. Lett. 77, 708 (2000). 